\title{
Microwave-induced Hall resistance in bilayer electron systems
}

\author{
S. Wiedmann,,${ }^{1,2,3}$ G. M. Gusev, ${ }^{4}$ O. E. Raichev,${ }^{5}$ S. Krämer, ${ }^{2}$ A. K. Bakarov, ${ }^{6}$ and J. C. Portal ${ }^{2,3}$ \\ ${ }^{1}$ Radboud University Nijmegen, Institute for Molecules and Materials, High Field Magnet Laboratory, \\ Toernooiveld 7, 6525 ED Nijmegen, The Netherlands \\ ${ }^{2}$ Laboratoire National des Champs Magnétiques Intenses, CNRS-UJF-UPS-INSA, F-38042 Grenoble, France \\ ${ }^{3}$ INSA Toulouse, F-31077 Toulouse Cedex 4, France \\ ${ }^{4}$ Instituto de Física da Universidade de São Paulo, CP 66318, São Paulo, SP, Brazil \\ ${ }^{5}$ Institute of Semiconductor Physics, NAS of Ukraine, Prospekt Nauki 41, 03028 Kiev, Ukraine \\ ${ }^{6}$ Institute of Semiconductor Physics, Novosibirsk 630090, Russia
}

(Received 10 February 2011; published 13 May 2011)

\begin{abstract}
The influence of microwave irradiation on dissipative and Hall resistance in high-quality bilayer electron systems is investigated experimentally. We observe a deviation from odd symmetry under magnetic-field reversal in the microwave-induced Hall resistance $\Delta R_{x y}$, whereas the dissipative resistance $\Delta R_{x x}$ obeys even symmetry. Studies of $\Delta R_{x y}$ as a function of the microwave electric field and polarization exhibit a strong and nontrivial power and polarization dependence. The obtained results are discussed in connection to existing theoretical models of microwave-induced photoconductivity.
\end{abstract}

DOI: 10.1103/PhysRevB.83.195317

PACS number(s): 73.40.-c, 73.21.-b, 73.43.-f

\section{INTRODUCTION}

In the past decade, it has been found that an external ac field [microwaves (MW's)] causes the appearance of microwave-induced resistance oscillations (MIRO's), ${ }^{1}$ which evolve into zero-resistance states (ZRS) for high-quality twodimensional electron systems (2DES) in the presence of a perpendicular magnetic field. ${ }^{2}$ MIRO's and ZRS occur in dissipative resistance but are not accompanied by plateaus in Hall resistance as for the integer quantum Hall effect. ${ }^{3}$ MIRO periodicity is governed by the ratio of radiation frequency $\omega$ to cyclotron frequency $\omega_{c}=e B / m$, where $m$ is the effective mass of the electrons. In theory, it is currently assumed that these oscillating phenomena can be explained by mechanisms originating from the scattering-assisted electron transitions between different Landau levels (LL's) in the presence of microwave excitation. The two main competing microscopic mechanisms for oscillating photoresistance are the "displacement" mechanism, which accounts for spatial displacement of electrons along the applied dc field under scattering-assisted microwave absorption, ${ }^{4,5}$ and the "inelastic" mechanism, owing to an oscillatory contribution to the isotropic part of the electron distribution function. ${ }^{6,7}$ Such a consideration describes the periodicity and phase of MIRO's observed in experiments.

Recently, it has been demonstrated that MW-induced phenomena in 2DES are not restricted to single-layer 2DES. Microwave-induced resistance oscillations have been found in bilayer and trilayer systems, ${ }^{8,9}$ and high-mobility bilayers with two occupied 2D subbands exhibit ZRS. ${ }^{10}$ The specific features in magnetoresistance in bilayers and multilayers are caused by an interference of magneto-intersubband (MIS) oscillations ${ }^{11}$ with MIRO's, when MW irradiation enhances, suppresses, or inverses the MIS oscillations.

Apart from dissipative resistance, one can ask if and how MW irradiation does affect Hall resistance since it was first a surprise, see Mani et al. in Ref. 2, that the Hall effect seemed to be unaffected by microwaves. Subsequent experiments on high-quality single-layer 2DES have shown weak
MW-induced oscillations in Hall resistance. ${ }^{12,13}$ The MWinduced Hall resistance $\Delta R_{x y}=R_{x y}-R_{x y}^{(0)}$, where $R_{x y}^{(0)}$ is the dark Hall resistance, depends on MW power and follows $1 / B$ periodicity of photoresponse in dissipative resistance. The observed odd symmetry under field reversal, $\Delta R_{x y}(B)=$ $-\Delta R_{x y}(-B)$, is preserved under increasing MW power. The studies in Refs. 12 and 13 have revealed basic information about MW-induced Hall resistance, though the role of microscopic mechanisms still remains unclear.

Theoreticians, however, have started to work on MWinduced Hall resistance, suggesting several microscopic mechanisms that describe how $\Delta R_{x y}$ is affected by an ac field. ${ }^{7,14}$ Dissipative resistivity $\rho_{x x}(B)=\rho_{x x}(-B)$, whose change at low temperatures is governed mostly by the inelastic mechanism, ${ }^{6,7}$ remains an even function under magneticfield reversal. In contrast, the mechanisms responsible for Hall resistivity lead to both odd- and even-symmetry contributions in $\Delta R_{x y}$. The presence of even-symmetry terms was discussed ${ }^{7,15}$ in connection with the important question of violation of Onsager-Casimir relations. ${ }^{16,17}$ Indeed, since MW-excited electron systems are far from thermodynamic equilibrium conditions, it is quite possible that the symmetry of the resistivity tensor is essentially broken under MW irradiation. Further experimental investigations of MW-induced Hall resistance are desirable to gain more knowledge about mechanisms of MW photoresistance and related symmetry properties of resistivity.

In this work, we have carried out measurements of MWinduced Hall resistance in high-mobility bilayers formed in wide quantum wells (WQW's) with high electron density. Due to charge redistribution in WQW's, there are two layers near the interfaces, separated by an electrostatic potential barrier, which create a symmetric tunnel-coupled bilayer electron system with two populated 2D subbands closely spaced in energy. ${ }^{10}$ Despite a complex photoresponse in bilayer systems, the smaller period of MIS oscillations ${ }^{11}$ compared to the MIRO period permits us a direct visualization of the quantum component of magnetoresistance that is affected by 
microwaves. This fact might be considered as an experimental advantage compared to a 2DES with only one occupied subband. ${ }^{18}$ We show $\Delta R_{x x}$ and $\Delta R_{x y}$ for both directions of the perpendicular magnetic field $B$ and demonstrate that MWinduced Hall resistance exhibits an MIS/MIRO interference with a strong deviation from odd symmetry under field reversal. In addition, we find strong and nontrivial power and polarization dependences of $\Delta R_{x y}$.

The paper is organized as follows. Section II presents experimental details on our samples and the experimental setup. Section III shows the results of photoresistance measurements including experiments where we have studied the dependence of photoresistance on the orientation of linear polarization. A discussion of the results in connection with theoretical models of MW-induced photoresistance in 2DES is presented in Sec. IV. Concluding remarks are given in the final section.

\section{EXPERIMENTAL DETAILS}

Our samples are high-quality WQW's, see Ref. 10, with a well width of $45 \mathrm{~nm}$, high electron density $n_{s} \simeq 9.1 \times$ $10^{11} \mathrm{~cm}^{-2}$, and a mobility of $\mu \simeq 1.9 \times 10^{6} \mathrm{~cm}^{2} / \mathrm{V} \mathrm{s}$ at $T=1.4 \mathrm{~K}$ after a brief illumination with a red light-emitting diode. The samples have Hall-bar geometry (length $l \times$ width $w=500 \mu \mathrm{m} \times 200 \mu \mathrm{m})$ with six contacts. In our experiment, we have used both linear and indeterminate polarization (frequency range 35-170 GHz). Microwave irradiation is delivered in a circular waveguide down to the sample placed in a cryostat with a variable temperature insert. To control linear polarization of MW's, we employ special brass insets that reduce the transmission-line internal profile from the circular to a rectangular waveguide and vice versa. The insets are placed on both sides of the circular waveguide to control orientation of the MW field vector for linear polarization. In the case of indeterminate polarization, the inset close to the sample is replaced by a circular extension of the waveguide, which implies that we still have linear polarization (of the amount $\simeq 90 \%$ ) but the orientation of the field vector is unknown. We measure $\mathrm{MW}$-induced resistance in a single-modulation ( $\mathrm{sm}$ ) technique and/or a double-modulation (dm) technique for a direct measurement of photoresponse to improve the measurement resolution. The bias current is $1 \mu \mathrm{A}$. In the sm technique, the sample is exposed to a continuous MW irradiation and a voltage drop is measured between two voltage probes at a frequency of $13 \mathrm{~Hz}$. In the dm technique, however, the MW's that are absorbed by the sample are amplitude modulated with an additional frequency of $333 \mathrm{~Hz}$. This enables us to directly probe $\Delta R$. To probe symmetry of $\Delta R_{x x}$ and $\Delta R_{x y}$ under field reversal, we have used two samples in Hall bar geometry that demonstrate the best symmetry of MIS and Shubnikov-de Haas ( $\mathrm{SdH}$ ) oscillations for low- and high-field transport without MW excitation.

\section{PHOTORESISTANCE MEASUREMENTS}

We start the presentation of our experimental results for a MW frequency of $143 \mathrm{GHz}$. In Fig. 1(a), we show first dark (no MW) normalized magnetoresistance $R_{x x}(B) / R_{x x}(0)$ and Hall resistance $R_{x y}$. The two-subband nature of our bilayer electron systems is confirmed by the presence of
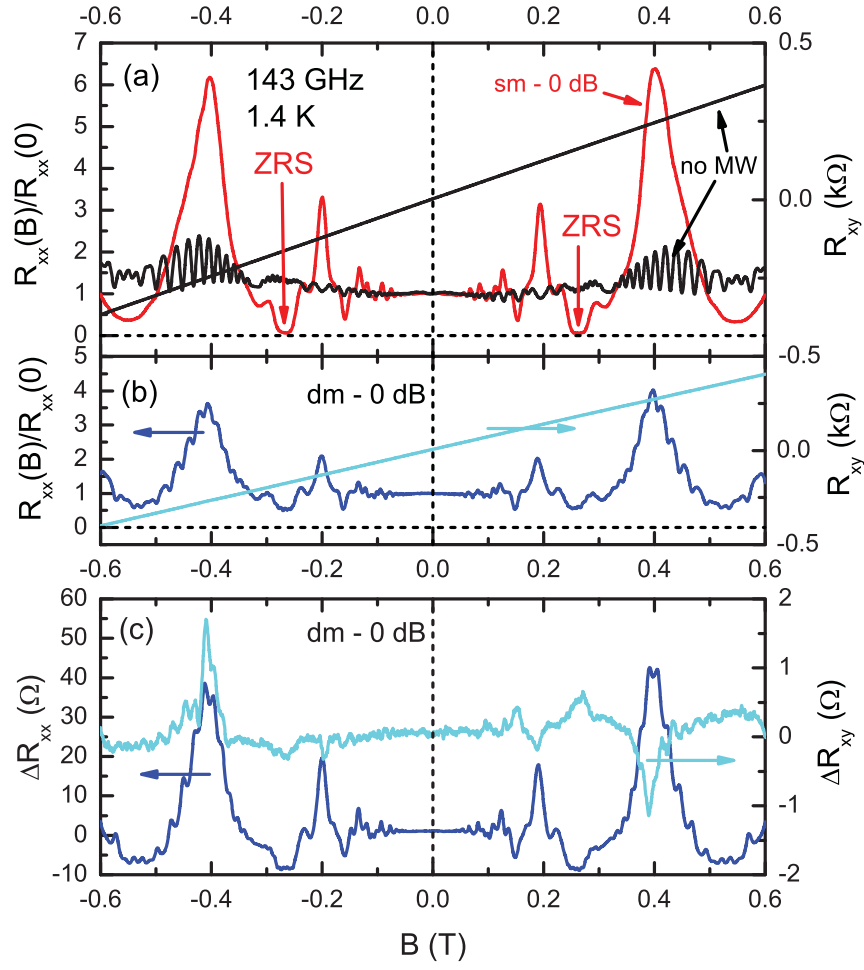

FIG. 1. (Color online) (a) Normalized resistance $R_{x x}(B) / R_{x x}(0)$ under microwave irradiation of $143 \mathrm{GHz}$ at $1.4 \mathrm{~K}$ for $0 \mathrm{~dB}$ attenuation (sm technique) as well as dark magnetoresistance (no MW) and Hall resistance $R_{x y}$. In $R_{x x}(B) / R_{x x}(0)$, we observe a ZRS at \pm 0.27 T. (b) $R_{x x}(B) / R_{x x}(0)$ and $R_{x y}$ for an attenuation of $0 \mathrm{~dB}$ (dm technique). (c) Photoresistance $\Delta R_{x x}$ and $\Delta R_{x y}$ measured in $\mathrm{dm}$ technique. We find an odd symmetry of $\Delta R_{x y}$ under field reversal.

MIS oscillations, which occur for $|B|>0.1 \mathrm{~T}$ and are superimposed on $\mathrm{SdH}$ oscillations at $1.4 \mathrm{~K}$. If we apply a MW electric field with $0 \mathrm{~dB}$ attenuation at a frequency of $143 \mathrm{GHz}$ in the sm technique, we observe ZRS for both negative and positive $B$ at $B= \pm 0.27 \mathrm{~T}^{10}$

The dissipative resistance in the $\mathrm{dm}$ technique does not show ZRS at $B= \pm 0.27$ T owing to a loss in MW power due to the modulation; see Fig. 1(b). The difference is seen in a smaller amplitude of enhanced MIS oscillations, see the peaks at \pm 0.4 and $\pm 0.2 \mathrm{~T}$, and also confirmed by the appearance of $\mathrm{SdH}$ oscillations for $|B|>0.4 \mathrm{~T}$. However, the electric field is strong enough to investigate the MW influence on our 2DES by a direct measurement of $\Delta R$, which will be used for further investigations. Photoresistance $\Delta R_{x x}$ and $\mathrm{MW}$-induced Hall resistance $\Delta R_{x y}$ measured directly in the dm technique are shown in Fig. 1(c). The main features of the modified MIS oscillation pattern in $\Delta R_{x x}$, which is symmetric under field reversal, are seen also in $\Delta R_{x y}$, but whereas $\Delta R_{x x}$ obeys even symmetry under field reversal, we find odd symmetry in $\Delta R_{x y}$ (see the region of the enhanced MIS peak at $\pm 0.4 \mathrm{~T}$ and the ZRS region at $\pm 0.27 \mathrm{~T}$ ). The result of odd symmetry in $\Delta R_{x y}$ is consistent with MW-induced Hall resistance in single-layer 2DES. ${ }^{12,13}$ However, having a closer look at $\Delta R_{x y}$ around $\pm 0.2 \mathrm{~T}$, we find a weak feature that deviates from odd symmetry under field reversal. This warrants further investigation.

In Fig. 2, we illustrate the power dependence of photoresistance for $f=143 \mathrm{GHz}$ at $1.4 \mathrm{~K}$. Starting again with $\Delta R_{x x}$ in 

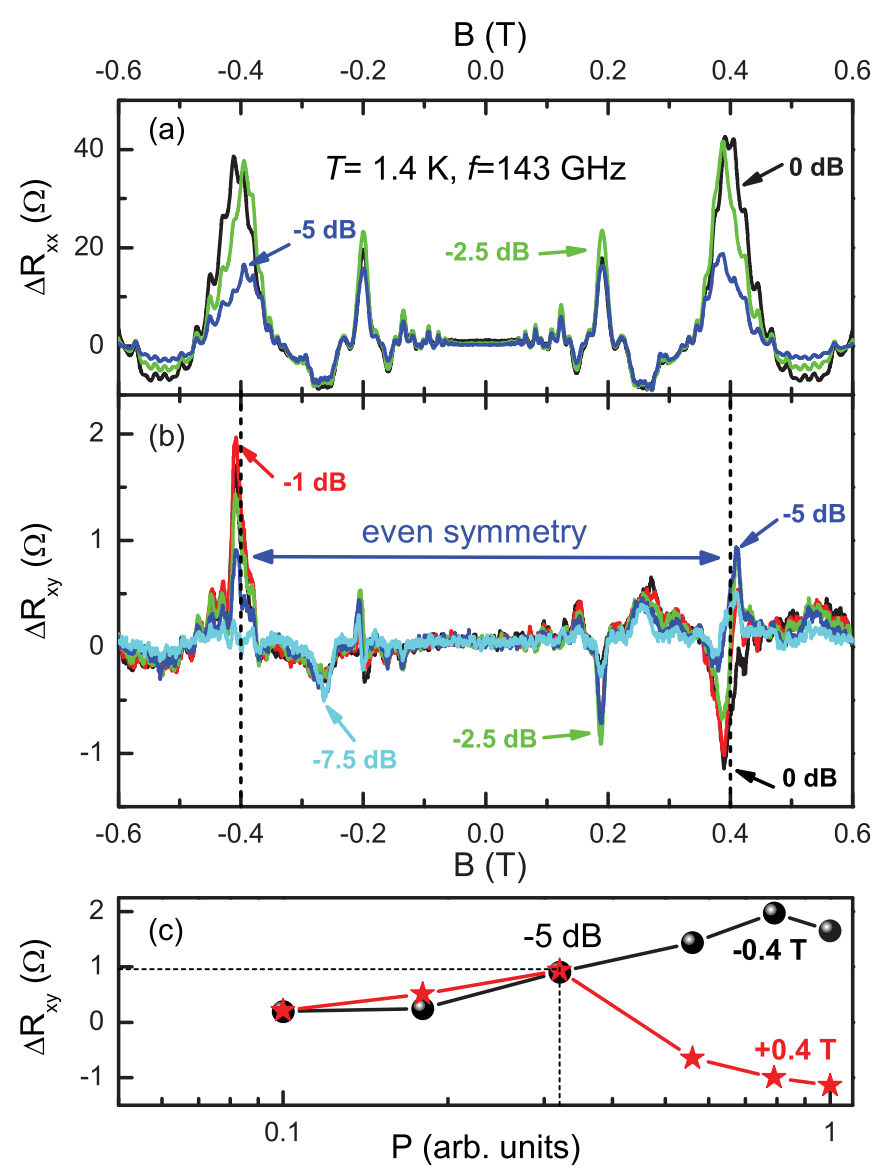

FIG. 2. (Color online) (a) Power-dependent $\Delta R_{x x}$ and (b) MWinduced Hall resistance at $143 \mathrm{GHz}$ and $1.4 \mathrm{~K}$. The enhanced resistance at $+0.4 \mathrm{~T}$ changes sign leading to an even symmetry for an attenuation of $-5 \mathrm{~dB}$. (c) $\Delta R_{x y}$ at $\pm 0.4 \mathrm{~T}$ as a function of MW power.

Fig. 2(a), we find that the amplitude decreases with decreasing MW power (attenuation from 0 to $-5 \mathrm{~dB}$ ) while preserving even symmetry under field reversal. However, the main peak of $\Delta R_{x y}$ at $+0.4 \mathrm{~T}$, see Fig. 2(b), changes its sign with decreasing MW power from 0 to $-7.5 \mathrm{~dB}$ (in contrast to the peak at $-0.4 \mathrm{~T}$ whose sign remains unchanged), so we find a transition from odd to even symmetry approximately at $-5 \mathrm{~dB}$. The Hall resistance (amplitude of the MIS peak) at $\pm 0.4 \mathrm{~T}$ is plotted as a function of MW power in Fig. 2(c). In contrast to this change in symmetry at $\pm 0.4 \mathrm{~T}$ with decreasing MW power, we notice that all other features in $\Delta R_{x y}$, e.g., at \pm 0.2 and $\pm 0.27 \mathrm{~T}$, do not exhibit an apparent change in symmetry. In addition, we find that with increasing temperature from 1.4 to $4 \mathrm{~K}$ (not shown here), only the amplitude of MIS oscillations in $\Delta R_{x y}$ decreases whereas the symmetry of $\Delta R_{x y}$ is preserved. This observation can be considered as an indication of the temperature independence of microscopic mechanisms contributing to MW-induced Hall resistance.

Whereas the inversion of one particular feature in Hall resistance leading to breaking of odd symmetry under field reversal has been observed at high frequencies, numerous power-dependent measurements of $\Delta R_{x y}$ for $f<75 \mathrm{GHz}$ demonstrated that several features in $\Delta R_{x y}$ for both negative and positive magnetic field change their signs in a nontrivial way as power varies. As an example of this behavior, we
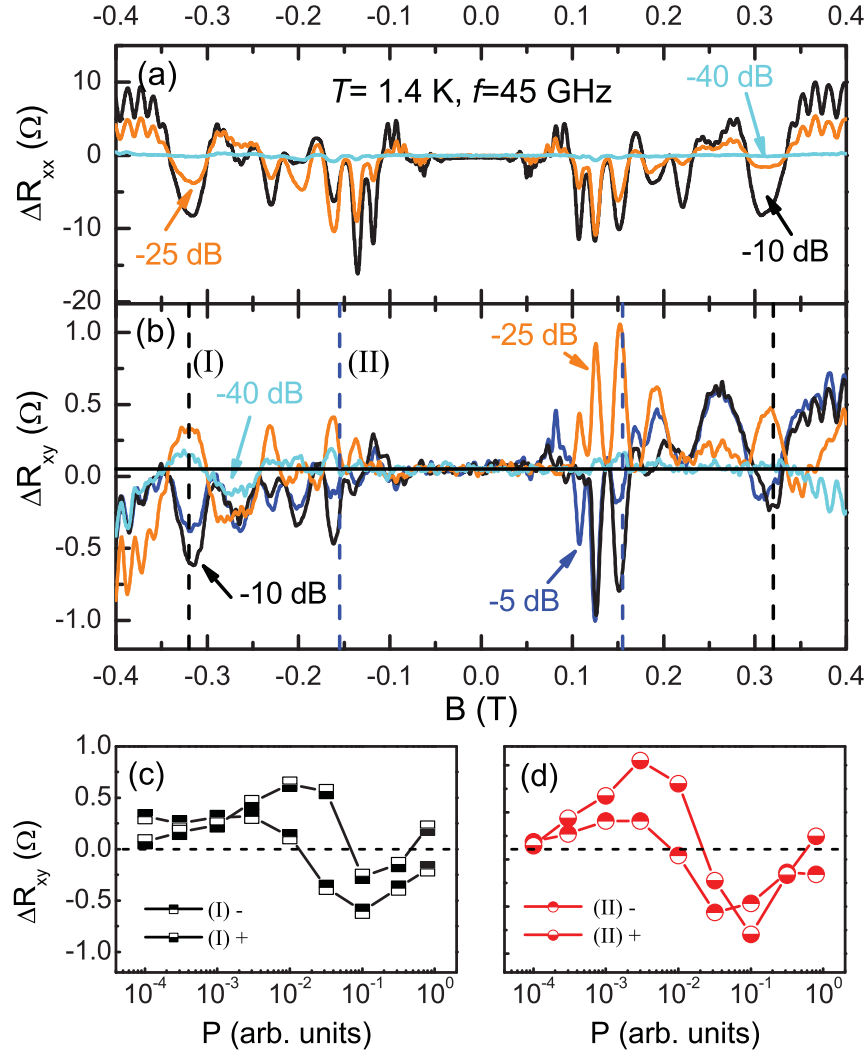

FIG. 3. (Color online) (a) Power dependence of $\Delta R_{x x}$ and (b) $\Delta R_{x y}$ for different chosen attenuations at $45 \mathrm{GHz}$ and $1.4 \mathrm{~K}$. For that frequency, $\Delta R_{x y}$ exhibits power-dependent MW-induced Hall resistance, denoted with peaks (I) and (II), which change their sign giving rise to odd or even symmetry. $\Delta R_{x y}$ in peak (I) (c) and in peak (II) (d) for $\pm B$ as a function of MW power.

present the power dependence of both $\Delta R_{x x}$ and $\Delta R_{x y}$ for $45 \mathrm{GHz}$ at $1.4 \mathrm{~K}$ in Fig. 3. Due to the possibility of our microwave setup, we investigate photoresistance at elevated MW power (see the estimates of the MW electric field in Sec. IV).

As a first impression, in Fig. 3(b) we see several MIS oscillations changing sign with decreasing MW power. These main features correlate with the MW response in $\Delta R_{x x}$; see Fig. 3(a). It should be noticed that for lower MW frequencies, see also Ref. 8, MIS oscillations show a more complicated behavior compared to high frequencies where several MIS peaks are strongly enhanced but they can be successfully described by the model used in Ref. 8 . Nevertheless, we get a reasonable even symmetry in $\Delta R_{x x}$ under field reversal, although MIS oscillations around $\pm 0.16 \mathrm{~T}$ differ in amplitude. Let us now focus on MW response in $\Delta R_{x y}$ in Fig. 3(b). For a better analysis, we mark two MIS oscillation peaks in $\Delta R_{x y}$ for $\pm B$ in Fig. 3(b) and denote them as (I) and (II). A change in sign of MW-induced Hall resistance occurs with decreasing MW power (from -5 to $-40 \mathrm{~dB}$ ) for all peaks except the one at $\pm 0.26 \mathrm{~T}$, where odd symmetry persists with changing attenuation. In particular, for $0<B<$ $0.15 \mathrm{~T}$ we observe several MIS oscillations whose amplitude is strongly enhanced by microwaves and which are inverted with decreasing MW power (compare data for -5 and $-25 \mathrm{~dB}$ attenuation). However, such a behavior with a comparable amplitude of MIS peaks is not observed for $-0.15<B<$ 
0 T. In summary, Hall resistance at $45 \mathrm{GHz}$ excitation obeys neither odd nor even symmetry. We now look more closely at the peaks marked with (I) and (II), for which we plot $\Delta R_{x y}$ as a function of MW power in Figs. 3(c) and 3(d). The equal sign of $\Delta R_{x y}$ for positive and negative $B$ can be considered as an indication of even symmetry with respect to field reversal. Starting with peak (I) at $\pm 0.32 \mathrm{~T}$, see Fig. 3(c), we always find even symmetry except for an attenuation of $-1 \mathrm{~dB}$ and around $-15 \mathrm{~dB}$. For peak (II) at $\pm 0.155 \mathrm{~T}$, we see a similar behavior, i.e., even symmetry is preserved with changing MW attenuation, except for a narrow region where peak flips occur. It is worth noting that all peak flips in MW-induced Hall resistance appear in the regions of MW power and magnetic field where MW's strongly affect the photoresistance $\Delta R_{x x}$.

We have also performed measurements where we control the linear polarization, i.e., the orientation of the MW electricfield vector $\mathbf{E}_{\omega}$. Due to a loss in MW power with brass insets, we carry out measurements in the sm technique and extract $\Delta R_{x y}$. We focus here on an intermediate frequency of $f=100 \mathrm{GHz}$. This frequency has been chosen because (i) the rectangular output of the brass inset is large enough to ensure a high enough MW electric field estimated to $E_{\omega} \simeq 1.5 \mathrm{~V} / \mathrm{cm}$ for $0 \mathrm{~dB}$ attenuation (this is not the case for $f>100 \mathrm{GHz}$ due to other brass insets), and (ii) a complicated power dependence for a fixed polarization, as for the case of $45 \mathrm{GHz}$, is avoided. Another argument in favor of $f=100 \mathrm{GHz}$ is the presence of one strongly enhanced MIS peak in $R_{x x}$ at $\pm 0.27 \mathrm{~T}$ and the corresponding prominent feature in $\Delta R_{x y}$, see Figs. 4(a) and 4(b), whose behavior is convenient to follow. The orientation of the electric field, i.e., tilt angle with respect to current direction, is also sketched for the angles $\Theta=0^{\circ}, 45^{\circ}$, and $90^{\circ}$ in Fig. 4. MW-induced Hall resistance $\Delta R_{x y}$ is measured at the highest possible MW power (close to $0 \mathrm{~dB}$ attenuation) at $1.4 \mathrm{~K}$, shown in steps of $\Delta \Theta=9^{\circ}$ in Fig. 4(b). To ensure the same MW electric field for all the tilt angles, we have compared the amplitude of $\mathrm{SdH}$ oscillations in $R_{x x}$ under the same conditions and found that it remains constant. ${ }^{8}$ Whereas $R_{x x}$ (and thus $\Delta R_{x x}$ ) does not depend on linear polarization, ${ }^{8-10,18} \mathrm{MW}$ induced Hall resistance exhibits essential angular dependence. If we focus on the enhanced peak in $R_{x x}$ at $\pm 0.27 \mathrm{~T}, \Delta R_{x y}$ shows even symmetry for, e.g., $\Theta=18^{\circ}$ and odd symmetry is observed for, e.g., $\Theta=54^{\circ}$. This is also illustrated in Fig. 4(c), where we plot the amplitude of $\Delta R_{x y}$ as a function of the angle between the current $\mathbf{I}$ and electric field $\mathbf{E}_{\omega}$ indicating a somehow oscillating behavior with increasing tilt angle. This result strongly indicates that in contrast to $\Delta R_{x x}, \Delta R_{x y}$ is sensitive to linear polarization of incident MW radiation and, consequently, microscopic mechanisms that account for $\Delta R_{x y}$ depend on the orientation of linear polarization.

\section{DISCUSSION}

Our experiments have undoubtedly shown that MW irradiation affects Hall resistance depending on MW power and orientation of linear polarization. Compared to power-dependent $\Delta R_{x y}$ in single-layer systems with a mobility of $1.5 \times 10^{7}$ $\mathrm{cm}^{2} / \mathrm{V}$ s, see Ref. 13, where a progressively stronger modulation of MIRO's is observed with increasing MW intensity, we demonstrate that our systems behave differently. The Hall resistance oscillations neither show that progressive increase with power nor obey the odd symmetry observed in Ref. 13 .
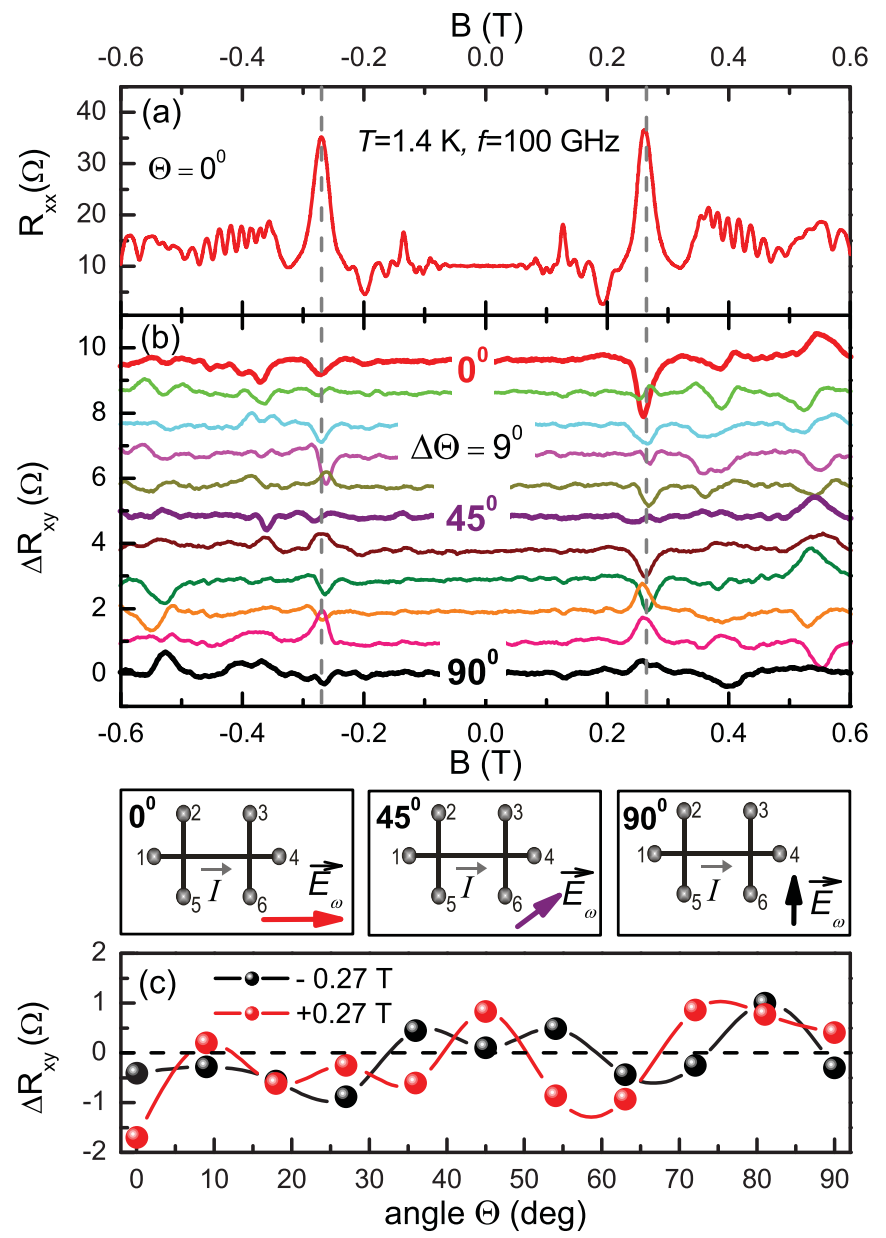

FIG. 4. (Color online) (a) $R_{x x}$ for $\Theta=0^{\circ}$ and (b) MW-induced Hall resistance $\Delta R_{x y}$ for $100 \mathrm{GHz}$ at $1.4 \mathrm{~K}$ dependent on the orientation of linear polarization from $\Theta=0^{\circ}$ to $90^{\circ}$, in steps of $\Delta \Theta=9^{\circ}$. (c) $\Delta R_{x y}$ at $\pm 0.27 \mathrm{~T}$ as a function of orientation of the electric field (tilt angle with respect to current direction-see sketches) exhibits alternating sign leading to odd (e.g., $\Theta=54^{\circ}, 90^{\circ}$ ) and even (e.g., $\Theta=18^{\circ}, 81^{\circ}$ ) symmetry. Orientation of the vector $\mathbf{E}_{\omega}$ is depicted in sketches for $\Theta=0^{\circ}, 45^{\circ}$, and $90^{\circ}$.

In that context, we first discuss existing theoretical models based on bulk mechanisms of photoconductivity. ${ }^{7,14,15}$ Considering a 2DES in the $(x y)$ plane, and assuming a linear regime of dc response to the applied field $\mathbf{E}$, one can write a conventional expression for the current density, $\mathbf{j}=\hat{\sigma} \mathbf{E}$, where the conductivity tensor in the $(x y)$ space is written as

$$
\hat{\sigma}=\left(\begin{array}{cc}
\sigma_{D}+\delta \sigma_{D s}+\delta \sigma_{D a} & -\sigma_{H}+\delta \sigma_{H s}+\delta \sigma_{H a} \\
\sigma_{H}+\delta \sigma_{H s}-\delta \sigma_{H a} & \sigma_{D}+\delta \sigma_{D s}-\delta \sigma_{D a}
\end{array}\right) .
$$

$\sigma_{D}$ and $\sigma_{H}$ are the dissipative and Hall conductivities in the absence of MW excitation, while $\delta \sigma_{D i}$ and $\delta \sigma_{H i}$ are the symmetric $(i=s)$ and antisymmetric $(i=a)$ MW-induced contributions to these conductivities. The resistivity tensor $\hat{\rho}$, defined according to $\mathbf{E}=\hat{\rho} \mathbf{j}$, is obtained directly from Eq. (1), and its nondiagonal component $\rho_{x y}$ is equal to the Hall resistance $R_{x y}$ :

$$
R_{x y}=\frac{\sigma_{H}-\delta \sigma_{H s}-\delta \sigma_{H a}}{\left(\sigma_{H}-\delta \sigma_{H a}\right)^{2}+\left(\sigma_{D}+\delta \sigma_{D s}\right)^{2}-\delta \sigma_{H s}^{2}-\delta \sigma_{D a}^{2}} .
$$


Below the onset of the quantum Hall effect, the dark Hall resistance $R_{x y}^{(0)}=\sigma_{H} /\left(\sigma_{H}^{2}+\sigma_{D}^{2}\right)$ is very close to the classical Hall resistance $R_{H}=B / e n_{s}$.

According to Refs. 6 and 7, the main photoinduced contribution to the dissipative conductivity is $\delta \sigma_{H s}$, caused (at low temperatures) mostly by the inelastic mechanism. The contributions $\delta \sigma_{D a}, \delta \sigma_{H s}$, and $\delta \sigma_{H a}$ are determined by the other three mechanisms called the displacement, the photovoltaic, and the quadrupole ones. ${ }^{7,15}$ Since these contributions are much smaller than $\sigma_{D}+\delta \sigma_{D s}$, one can rewrite Eq. (2) as

$$
\begin{gathered}
R_{x y}=R_{H}+\rho_{x y}^{(1)}+\rho_{x y}^{(2)}, \quad \rho_{x y}^{(1)}=\frac{\delta \sigma_{H a}-\delta \sigma_{H s}}{\sigma_{H}^{2}}, \\
\rho_{x y}^{(2)}=-\frac{\left(\sigma_{D}+\delta \sigma_{D s}\right)^{2}}{\sigma_{H}^{3}},
\end{gathered}
$$

where it is also taken into account that $\sigma_{D}+\delta \sigma_{D s} \ll \sigma_{H}$ and $\sigma_{H} \simeq 1 / R_{H}$, which assumes a finite (not very small) magnetic field.

Thus, the MW-induced modification of Hall resistance is given by two terms. The first one is determined directly by the Hall photoconductivity contributions $\delta \sigma_{H a}$ and $\delta \sigma_{H s}$, while the second one is related to dissipative resistivity. The theory ${ }^{7,15}$ attributes $\delta \sigma_{\mathrm{Ha}}$ and $\delta \sigma_{\mathrm{HS}}$ to the contributions of the photovoltaic and quadrupole mechanisms, respectively. Both of these contributions retain odd symmetry under magnetic-field reversal, though the presence of $\delta \sigma_{H s}$ leads to a violation of the relation $\sigma_{x y}(B)=\sigma_{y x}(-B)$. The nature of the quadrupole mechanism suggests that $\delta \sigma_{H s}$ is polarizationdependent. However, the consideration in Refs. 7 and 15 is valid for elliptical polarization of a MW field in the main axes $(x y)$ and can be applied for linear polarization along either the $x$ or $y$ axis $\left(\Theta=0^{\circ}\right.$ or $\left.90^{\circ}\right)$. The case of arbitrary linear polarization is studied in Ref. 14 by considering the displacement mechanism of photoconductivity. It was shown that the corresponding $\delta \sigma_{H s}$ contains a contribution that is an even function of the magnetic field. This contribution depends on the tilt angle as $\sin (2 \Theta)$. In summary, the term $\rho_{x y}^{(1)}$ can lead to an even-symmetry part of the Hall resistivity tensor, and this part is polarization-dependent.

In contrast, the term $\rho_{x y}^{(2)}$ possesses an odd symmetry under field reversal and is polarization-independent. As a first impression, this term should be less significant, because of strong inequality $\sigma_{D}+\delta \sigma_{D s} \ll \sigma_{H}$. However, another strong inequality, $\left|\delta \sigma_{D s}\right| \gg\left|\delta \sigma_{H a} \pm \delta \sigma_{H s}\right|$, appears to be more important, and our estimates prove that $\rho_{x y}^{(2)}$ is the main part of the Hall resistance under our experimental conditions. Specifically, we have applied theoretical expressions ${ }^{7}$ for $\delta \sigma_{H a}$ and $\delta \sigma_{H s}$ with known MW field $E_{\omega} \simeq 2 \mathrm{~V} / \mathrm{cm}$ (0 $\mathrm{dB}$ attenuation) for $f=143 \mathrm{GHz}$ and $E_{\omega} \simeq 3 \mathrm{~V} / \mathrm{cm}$ ( $-10 \mathrm{~dB}$ attenuation) for $f=45 \mathrm{GHz}$, and we found that $\rho_{x y}^{(1)}$ is approximately one order of magnitude smaller than $\rho_{x y}^{(2)}$ (and even smaller for $f=143 \mathrm{GHz}$ ). A similar conclusion is made in Ref. 14 by proving that at $\Theta=45^{\circ}$, when the even-symmetry part of the Hall resistivity tensor is maximal, this part still does not produce an appreciable deviation from the odd symmetry. Therefore, the main contribution to the Hall resistivity comes from $\rho_{x y}^{(2)}$ and the odd symmetry should be preserved. The importance of the term $\rho_{x y}^{(2)}$ is also emphasized in Ref. 19.

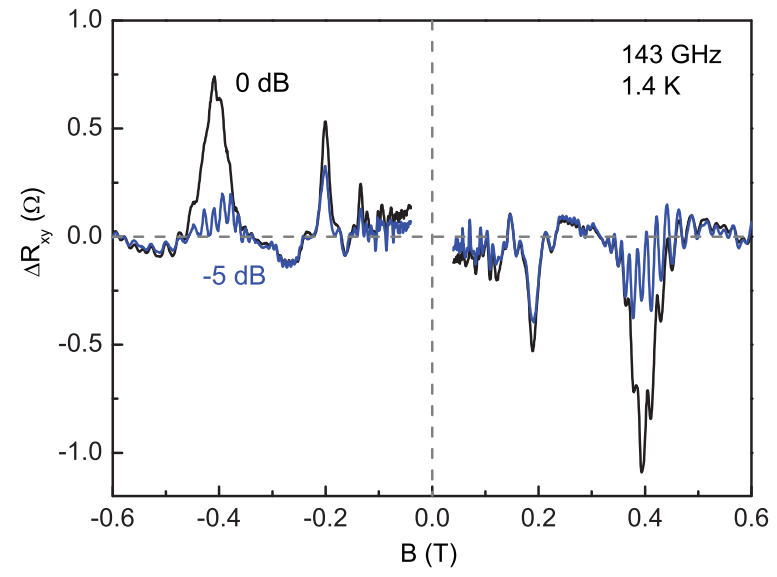

FIG. 5. (Color online) MW-induced Hall resistance $\Delta R_{x y}$ for 143 $\mathrm{GHz}(0$ and $-5 \mathrm{~dB}$ attenuation) and $1.4 \mathrm{~K}$ calculated from Eq. (4).

By retaining only $\rho_{x y}^{(2)}$ in Eq. (3), one can rewrite the MWinduced Hall resistivity in the form

$$
\Delta R_{x y} \simeq \frac{\rho_{x x}^{(0) 2}-\rho_{x x}^{2}}{R_{H}},
$$

where $\rho_{x x}^{(0)}$ is the dark dissipative resistivity. This relation does not contain the details of photoconductivity mechanisms and can be checked directly by using the dissipative resistivity $\rho_{x x}$ measured in the experiment. We have carried out such a procedure for both 143 and $45 \mathrm{GHz}$ and different attenuations. The results for $143 \mathrm{GHz}$ are presented in Fig. 5. One can see that the amplitudes of the main peaks at \pm 0.2 and $\pm 0.4 \mathrm{~T}$ are close to those obtained experimentally and behave in a similar way with decreasing power. The sign inversion of $\Delta R_{x y}$ just above $+0.4 \mathrm{~T}$, leading to apparent reversal of symmetry from odd to even for $-5 \mathrm{~dB}$ attenuation, can be explained in terms of a slight asymmetry of measured dissipative resistivity [see Fig. 2(a)] and alteration of the sign of $\rho_{x x}^{(0) 2}-\rho_{x x}^{2}$ in this region of magnetic field.

The amplitudes of the peaks in $\Delta R_{x y}$ for other MW frequencies used in the experiment are also in reasonable agreement with Eq. (4). However, neither the strong and complicated modifications of the Hall resistance with MW power at $45 \mathrm{GHz}$ (Fig. 3) nor the polarization dependence (Fig. 4) can be reproduced using this simple expression. In this connection, we again discuss the possible influence of the term $\rho_{x y}^{(1)}$, which is essentially determined by the Hall photoconductivity mechanisms. In principle, this term can become comparable with $\rho_{x y}^{(2)}$ if the field $E_{\omega}$ is considerably larger than that used in our estimates. A theoretical study in Ref. 20 suggests that the MW field in the near-contact regions of the Hall bar is strongly enhanced compared to the MW field in the bulk of the sample. Therefore, the possibility that electrons feel a stronger field should not be disregarded. The Hall photoconductivity mechanisms can lead to inversion of oscillation peaks in $\rho_{x y}^{(1)}$ under a transition to the regime when the oscillating nonequilibrium part of electron distribution saturates with increasing $E_{\omega}$. This saturation effect is discussed in detail in Refs. 6 and 7. However, our estimates show that at $45 \mathrm{GHz}$ and $-10 \mathrm{~dB}$ attenuation $\left(E_{\omega} \simeq 3 \mathrm{~V} / \mathrm{cm}\right)$, the $2 \mathrm{DEG}$ at $1.4 \mathrm{~K}$ is already in the saturation regime. The 
assumed increase of $E_{\omega}$ in the near-contact regions cannot, therefore, cause inversion of oscillations in $\Delta R_{x y}$ due to the saturation effect. Furthermore, if we look at the polarization dependence, the even contribution to the Hall resistivity in $\rho_{x y}^{(1)}$ should follow, according to the theory, a simple $\sin (2 \Theta)$ law. Instead, in Fig. 4 we see multiple oscillations of both even and odd contributions as functions of $\Theta$, and the even contribution does not disappear at $\Theta=0^{\circ}$ and $90^{\circ}$, contrary to the theoretical prediction. Therefore, even if we assume that the field $E_{\omega}$ is effectively enhanced, the complicated behavior of the observed MW-induced Hall resistance (Figs. 3 and 4) cannot be explained by employing the bulk mechanisms of MW photoconductivity.

An alternative approach to the MW-induced effects in dissipative resistance such as MIRO and ZRS was recently proposed in Ref. 21. It is suggested that these effects have a purely classical origin. They are induced by ponderomotive forces that arise in the near-contact regions because of a strong inhomogeneity of the MW field and possess an oscillatory dependence on MW frequency and magnetic field. It is not clear, however, whether the presence of such ponderomotive forces can contribute to the Hall resistance $\Delta R_{x y}$. In any case, it cannot lead to a polarization dependence of $\Delta R_{x y}$, because the MW polarization in the near-contact regions is fixed (the MW field is perpendicular to the boundary between 2DEG and contact) regardless of polarization of the incident wave. The nontrivial power dependence of $\Delta R_{x y}$ observed in our experiment cannot be explained within this approach as well.

Another approach to the MW-induced magnetotransport is developed in Ref. 22, where the influence of MW's on the edge trajectories has been studied and the appearance of ZRS is explained in terms of stabilization of the edge-state transport by MW's. A deviation of $R_{x y}$ from the classical Hall resistance, which correlates with the corresponding changes in the dissipative resistance $R_{x x}$, is also mentioned. Both $R_{x x}$ and $R_{x y}$ are found to be sensitive to the direction of linear polarization of MW's. The implication of these results to symmetry properties of $R_{x y}$ with respect to magnetic-field reversal has not been discussed. The theory of Ref. 22 might be relevant to the samples with very high mobilities, such as those studied in Ref. 13, where edge trajectories are still important for transport in the region of magnetic fields below $0.5 \mathrm{~T}$. Presumably, the edge-state transport in these samples is responsible for the fact that the oscillations of MW-induced Hall resistance $\Delta R_{x y}$ and the oscillations of $\rho_{x x}$ have comparable amplitudes, which also means that $\Delta R_{x y}$ cannot be described by Eq. (4). In our samples, however, the transport is expected to be bulklike (diffusive) in the mentioned region of magnetic fields, since Eq. (4) proves to be applicable for estimating the magnitude of MW-induced $\Delta R_{x y}$.

Finally, we cannot completely rule out the possibility that the complicated behavior of $\Delta R_{x y}$ is related to specific features of bilayer (two-subband) systems as compared to singlelayer systems. Above, we have stated that the only essential difference between magnetoresistances of single-subband and two-subband systems is the modulation of the quantum contribution to resistivity by the MIS oscillations in twosubband 2DES. This statement is well justified from the point of view of bulk transport theory and is confirmed in numerous experiments. ${ }^{8-11,18,23-25}$ In addition, the theoretical model of dissipative MW photoresistance based on a consideration of the inelastic mechanism ${ }^{6}$ explains satisfactorily all features of MIRO's (including frequency, power, and temperature dependence) for different two-subband systems studied in our experiments (see, e.g., Refs. 8 and 10). The MW-induced Hall resistance, however, is a subtle effect: in our sample, $\Delta R_{x y} \ll \Delta R_{x x}$. If the transport is influenced by the presence of sample edges or contact regions, the bilayer nature of our system may essentially manifest itself in $R_{x y}$. To check out this assumption, it is desirable to measure MW-induced $R_{x y}$ in single-layer 2DES whose density and mobility are close to those of our system.

\section{CONCLUSIONS}

We have studied the photoresponse of $\Delta R_{x x}$ and $\Delta R_{x y}$ in high-quality bilayer electron systems. Whereas even symmetry is preserved in $\Delta R_{x x}$ with a reasonable accuracy, we found a violation of odd symmetry in MW-induced Hall resistance, in contrast to previous experiments ${ }^{12,13}$ on single-layer 2DES with higher mobilities. A nontrivial power dependence is observed for several MIS oscillation peaks in $\Delta R_{x y}$. Symmetry of $\Delta R_{x y}$ is also essentially modified by changing MW power. Varying $\Delta R_{x y}$ for different orientations of linear polarization strongly confirms the feasibility of polarization-dependent microscopic mechanisms of MW-induced Hall resistance, in contrast to polarization immunity in dissipative resistance. ${ }^{8,10,26}$ The photoresponse in $\Delta R_{x y}$ might be accounted for by the presence of two components in $\Delta R_{x y}$, of which one is odd and another is even with respect to magnetic-field reversal. A reasonably good estimate for the magnitude of $\Delta R_{x y}$ is obtained within the bulk transport approach. However, bulk transport models, as well as currently existing alternative approaches to the problem of MW-induced resistance, fail to explain nontrivial power and polarization dependence and the strong violation of odd symmetry observed in our experiments. Due to the essential deviation of our data from the theoretical models discussed above, we cannot preclude the influence of possible previously unconsidered microscopic mechanisms that might exist in a broad interval of MW power or turn on at elevated MW power.

In general, our data and its analysis suggest that the problem of MW-induced Hall resistance (and, hence, the related problem of MW-induced dissipative resistance) still remains a puzzle that awaits a future solution. We suppose that a theory that could describe the behavior of both components of the MW-induced resistivity on an equal footing and explain the variety of experimental facts has to be based on a consideration of quantum transport in the presence of a strongly inhomogeneous MW field. We assume that our systematic study will stimulate further experimental and theoretical investigations, which are crucial to clarify the origin of MW-induced phenomena in 2DES.

\section{ACKNOWLEDGMENTS}

We acknowledge support from COFECUB-USP (project number $\mathrm{U}_{c} \mathrm{Ph}$ 109/08), FAPESP, and CNPq (Brazilian agencies). For measurements we used microwave facilities from ANR MICONANO. 
${ }^{1}$ M. A. Zudov, R. R. Du, J. A. Simmons, and J. L. Reno, Phys. Rev. B 64, 201311(R) (2001); P. D. Ye, L. W. Engel, D. C. Tsui, J. A. Simmons, J. R. Wendt, G. A. Vawter, and J. L. Reno, Appl. Phys. Lett. 79, 2193 (2001).

${ }^{2}$ R. G. Mani, J. H. Smet, K. von Klitzing, V. Narayanamurti, W. B. Johnson, and V. Umansky, Nature (London) 420, 646 (2002); M. A. Zudov, R. R. Du, L. N. Pfeiffer, and K. W. West, Phys. Rev. Lett. 90, 046807 (2003); R. L. Willett, L. N. Pfeiffer, and K. W. West, ibid. 93, 026804 (2004).

${ }^{3}$ K. v. Klitzing, G. Dorda, and M. Pepper, Phys. Rev. Lett. 45, 494 (1980).

${ }^{4}$ V. I. Ryzhii, Fiz. Tverd. Tela (Leningrad) 11, 2577 (1969) [Sov. Phys. Solid State 11, 2078 (1970)]; V. I. Ryzhii, R. A. Suris, and B. S. Shchamkhalova, Fiz. Tekh. Poluprovodn. 20, 2078 (1986) [Sov. Phys. Semicond. 20, 1299 (1986)].

${ }^{5}$ A. C. Durst, S. Sachdev, N. Read, and S. M. Girvin, Phys. Rev. Lett. 91, 086803 (2003).

${ }^{6}$ I. A. Dmitriev, M. G. Vavilov, I. L. Aleiner, A. D. Mirlin, and D. G. Polyakov, Phys. Rev. B 71, 115316 (2005).

${ }^{7}$ I. A. Dmitriev, A. D. Mirlin, and D. G. Polyakov, Phys. Rev. B 75, 245320 (2007).

${ }^{8}$ S. Wiedmann, G. M. Gusev, O. E. Raichev, T. E. Lamas, A. K. Bakarov, and J. C. Portal, Phys. Rev. B 78, 121301(R) (2008).

${ }^{9}$ S. Wiedmann, N. C. Mamani, G. M. Gusev, O. E. Raichev, A. K. Bakarov, and J. C. Portal, Phys. Rev. B 80, 245306 (2009).

${ }^{10}$ S. Wiedmann, G. M. Gusev, O. E. Raichev, A. K. Bakarov, and J. C. Portal, Phys. Rev. Lett. 105, 026804 (2010).
${ }^{11}$ N. C. Mamani, G. M. Gusev, T. E. Lamas, A. K. Bakarov, and O. E. Raichev, Phys. Rev. B 77, 205327 (2008).

${ }^{12}$ S. A. Studenikin, M. Potemski, P. T. Coleridge, A. S. Sachrajda, and Z. R. Wasilewski, Solid State Commun. 129, 341 (2004).

${ }^{13}$ R. G. Mani, V. Narayanamurti, K. von Klitzing, J. H. Smet, W. B. Johnson, and V. Umansky, Phys. Rev. B 69, 161306(R) (2004).

${ }^{14}$ V. I. Ryzhii, J. Phys. Soc. Jpn. 73, 1539 (2004).

${ }^{15}$ I. A. Dmitriev, M. Khodas, A. D. Mirlin, D. G. Polyakov, and M. G. Vavilov, Phys. Rev. B 80, 165327 (2009).

${ }^{16}$ L. Onsager, Phys. Rev. 38, 2265 (1931).

${ }^{17}$ H. B. G. Casimir, Rev. Mod. Phys. 17, 343 (1945).

${ }^{18}$ S. Wiedmann, G. M. Gusev, O. E. Raichev, A. K. Bakarov, and J. C. Portal, Phys. Rev. B 81, 085311 (2010).

${ }^{19}$ J. Iñarrea, Appl. Phys. Lett. 90, 172118 (2007).

${ }^{20}$ S. A. Mikhailov and N. A. Savostianova, Phys. Rev. B 74, 045325 (2006).

${ }^{21}$ S. A. Mikhailov, Phys. Rev. B 83, 155303 (2011).

${ }^{22}$ A. D. Chepelianskii and D. L. Shepelyansky, Phys. Rev. B 80, 241308(R) (2009).

${ }^{23}$ S. Wiedmann, G. M. Gusev, O. E. Raichev, A. K. Bakarov, and J. C. Portal, Physica E 42, 1075 (2010).

${ }^{24}$ A. A. Bykov, D. R. Islamov, A. V. Goran, and A. I. Toporov, JETP Lett. 87, 477 (2008).

${ }^{25}$ G. M. Gusev, S. Wiedmann, O. E. Raichev, A. K. Bakarov, and J. C. Portal, Phys. Rev. B 83, 041306(R) (2011).

${ }^{26}$ J. H. Smet, B. Gorshunov, C. Jiang, L. Pfeiffer, K. West, V. Umansky, M. Dressel, R. Meisels, F. Kuchar, and K. von Klitzing, Phys. Rev. Lett. 95, 116804 (2005). 